\title{
Collective jumps in a soft-sphere glass
}

\author{
C. Oligschleger* \\ Institut für Algorithmen und Wissenschaftliches Rechnen, GMD-Forschungszentrum Informationstechnik, \\ D-53754 St. Augustin, Germany \\ and Institut für Festkörperforschung, Forschungszentrum Jülich, D-52425 Jülich, Germany
}

H. R. Schober

Institut für Festkörperforschung, Forschungszentrum Jülich, D-52425 Jülich, Germany

(Received 5 February 1998; revised manuscript received 8 May 1998)

\begin{abstract}
Relaxations in a soft-sphere glass are studied by molecular dynamics. We observe local jumps where groups of ten and more atoms move collectively forming chainlike structures. At the lowest temperatures the total jump length is of the order of the nearest neighbor distance whereas a single atom moves only a fraction of this distance. Both the displacements and the numbers of participating atoms increase with temperature. Successive jumps tend to involve the same atoms. Relaxations and soft quasilocalized vibrations are correlated. In the glass and in the liquid state near the glass transition a markedly non-Gaussian distribution of the mean square displacements is observed. [S0163-1829(99)10601-5]
\end{abstract}

\section{INTRODUCTION}

The dynamics of glasses and amorphous materials is characterized by some distinct features. At the lowest frequencies one finds normal sound waves corresponding to the usual Debye behavior. At higher frequencies additional vibrational excitations are observed. These lead to the so-called Boson peak in the dynamic scattering function, typically around 1 THz. Additionally to these vibrational excitations which obey approximately Bose statistics, two level excitations are observed below a few K. ${ }^{1,2}$ These can be envisaged as quantum-mechanical tunneling transitions between adjacent glassy configurations of nearly equal energy. They dominate at the lowest temperatures properties such as specific heat, sound attenuation, and internal friction. A model description is provided by the standard tunneling model. ${ }^{3,4}$ In this temperature range glasses show a quasiuniversal behavior.

At higher temperatures experiments indicate the existence of wide distributions of relaxations. ${ }^{5-10}$ These can be envisaged as thermally activated transitions over barriers, again separating local minima in configurational space. These barriers will be higher, the configurations further apart and their energies more different than in the tunneling case. The properties of glasses vary much more between materials in this temperature regime. These "relaxations" from one local minimum into another can lead to diffusion or merely to local rearrangements. This depends on the interconnecting paths between the local minima in the configurational space. If one takes the grossly oversimplified picture of a multidimensional mountain range, representing the potential energy as function of all atomic positions, then for a given maximal altitude, representing the temperature, some minima will be connected while others cannot be reached. A mountaineer, our point in configurational space, can either be confined to a single minimum or a small number of minima or he can wander off to far away places. Since jumps over barriers are in general only weakly influenced by the structure of the second minima diffusional jumps are expected to be similar to nondiffusional ones.

A semiempirical description of the behavior up to temperatures of about $20 \mathrm{~K}$ was given by the soft potential model (SPM). ${ }^{11,12}$ A basic assumption of this model is a strong correlation between soft quasilocal vibrations (QLV) and local relaxations. A distribution of soft potentials for the movement of some reaction coordinate is assumed. Depending on the parameters these potentials can be either one well or two well, thus describing either QLV or, depending on the barrier height, tunneling or activated hopping. The modes described by these effective potentials coexist with the "normal" vibrational modes such as sound waves. This model encompasses the standard tunneling model as its lowtemperature limit. It does not, however, give direct insight into the nature of the modes. By fitting this model to the experimental data, one finds effective masses of 20-100 atomic masses for the entities moving in these effective soft potentials. ${ }^{13,14}$ The model can be extended to higher temperatures and energies using plausible assumptions on the distribution of the model parameters. ${ }^{15}$ The validity of the assumption of a strong correlation between the different excitations has been questioned recently. ${ }^{16}$

Computer simulations of the soft-sphere glass (SSG) confirmed the existence of low frequency quasilocalized vibrations with effective masses ranging from ten atomic masses upwards, Refs. 17 and 18. The modes were centered at structural irregularities with large local strains. Regions of local strain have also been observed in earlier computer simulations. ${ }^{19}$ Similar effective masses have since been seen in simulations of $\mathrm{SiO}_{2},{ }^{20} \mathrm{Se}$ (Ref. 21), in Ni-Zr, ${ }^{22} \mathrm{Pd}-\mathrm{Si}$, ${ }^{23}$ $\mathrm{Ni}-\mathrm{B},{ }^{24}$ in amorphous ice, ${ }^{25}$ and in amorphous and quasicrystalline $\mathrm{Al}-\mathrm{Zn}-\mathrm{Mg} .{ }^{26} \mathrm{In}$ an earlier simulation of amorphous silicon low frequency localized vibrations have been observed at coordination defects. ${ }^{27}$ It should be stressed that the notion of low frequency localized modes is an idealization. These modes will always interact with the sound waves and therefore also with each other. The interaction with these 
modes causes a damping of the sound waves which reaches the Ioffe-Regel criterium around the Boson peak. The mixing of the two types of modes becomes so strong that they are no longer distinguishable. The resulting modes can no longer be described as either quasilocal or as extended phonons similar to crystalline ones. To show their different behavior they have been called diffusons. ${ }^{28}$ The soft local vibrations of the soft potential model have to be understood as idealized modes with this interaction "switched off." ${ }^{29}$ Strong anharmonic effects in the QLV have been observed both in computer simulations and in experiment. ${ }^{30}$

Computer simulations of relaxations in amorphous structures are more difficult. One approach is to search the potential-energy hypersurface for adjacent minima. Heuer and Silbey ${ }^{31}$ searched systematically in a Lennard-Jones-type glass for pairs of local minima of the potential energy. A parametrization in terms of the soft potential model again gives a qualitative agreement with the assumptions of that model. The effective masses of the jump modes from one well into the other ranged from two atomic masses upwards.

Monitoring the atomic displacements in molecular dynamics simulations just above the glass transition in many different materials, single atom jumps over nearest neighbor distances have been observed, e.g., in a soft-sphere system ${ }^{32}$ and a two component Lennard-Jones system. ${ }^{33}$ In the strongly supercooled regime these jumps have been found to be strongly cooperative. In metallic systems one observes typically chains of jumping atoms. ${ }^{34-38}$ Additionally to these jumps a motion involving shorter distances per atom is seen in molecular dynamics which is mostly referred to as cage or stray motion. This motion does, however, contribute to diffusion. ${ }^{39}$

In the glassy state strongly collective jumps were observed in molecular dynamics studies at temperatures ranging up to 15 and $30 \%$ of the glass temperature for the SSG (Ref. 40) and for Se, ${ }^{41}$ respectively. In these jumps the total jump length, added over all atoms, is of the order of the atomic distance but, different from the above mentioned jumps in the liquid, the single atoms jump only a fraction of this distance. The numbers of atoms involved is similar to the one in the quasilocalized vibrations.

The mechanism of diffusion in metallic glasses is still highly controversial, for reviews see Refs. 42-44. Experiments are often interpreted in terms of the free volume model. ${ }^{45}$ In its most simple interpretation one assumes that an atom can jump to an adjacent site whenever this opens up due to fluctuations. Essentially this corresponds to a "singleatom-jump model." An alternative approach to the concept of a free volume was given in Ref. 46 in terms of holes which are more spread and where atoms surrounding the holes jump collectively.

Related to the free volume concept is the concept of diffusion via thermally created vacancies. Such a mechanism was, e.g., postulated for $\mathrm{Hf}$ diffusion in amorphous $\mathrm{Ni}_{54} \mathrm{Zr}_{46}$ where, from pressure experiments, an activation volume for diffusion of the order of the atomic volume was deducted ${ }^{47}$ similar to earlier results for Co diffusion. ${ }^{48}$ The opposite result was found for $\mathrm{Co}$ in $\mathrm{Co}_{81} \mathrm{Zr}_{19}$ where this mechanism was ruled out due to the vanishing activation volume. ${ }^{49}$ No pressure dependence of $\mathrm{Co}$ and $\mathrm{Fe}$ diffusion has been observed in the metal-metalloid glasses $\mathrm{Co}_{76.7} \mathrm{Fe}_{2} \mathrm{Nb}_{14.3} \mathrm{~B}_{7}$ and $\mathrm{Fe}_{40} \mathrm{Ni}_{40} \mathrm{~B}_{20}$ glasses. ${ }^{50-52}$

Another possibility of a defect mediated diffusion is via an interstitial mechanism. This will certainly hold for diffusion of small atoms such as $\mathrm{H}$ (Ref. 53) but has also been assumed for larger atoms such as Co. ${ }^{48}$

The very small isotope effect observed experimentally ${ }^{50,54}$ points towards a diffusion mechanism inherent to the disordered structure. This would be in agreement with the collective jumps seen in the simulation. ${ }^{40}$

The situation is complicated by the large aging effects in amorphous metals. The above results refer to "fully relaxed" materials. In unrelaxed materials a tendency to single atom jumps is observed. ${ }^{55,43}$ However, there are indications that in relaxed metallic glasses at least the structure-forming component diffuses by a collective mechanism intrinsic to the disorder. In alloys, particularly for small atoms, different mechanisms might be expected. Our computer study of a one-atomic metallic glass should shed some light on the properties of these jumps without the complications introduced by the experimentally necessary alloying.

In this paper we present a comprehensive report of our investigation of relaxations in a SSG at low and moderate temperatures. The first aim is to get some insight into the structural relaxations in a glass as such. To extend the model description beyond the "universal" low-temperature regime the validity of the assumption of correlations between vibrations and relaxations needs to be tested, and the change with temperature studied. This can best be done with a simple model glass which has, other than the hard sphere glass, well defined phonons at all temperatures. Learning about the single relaxations will give insight into the diffusion mechanism at these temperatures. A direct observation of diffusion is of course ruled out by the orders of magnitude higher computer time needed.

We describe the soft-sphere glass by a purely repulsive $1 / R^{6}$ interaction. This interaction is much softer than the often taken $1 / R^{12}$ law. The exponent is chosen to be near the phase transitions between hcp, fcc, and bcc crystalline phases. Whether the fcc or hcp phase is the ground state depends critically on the cutoff procedure and length. For our choice the hcp structure was found to be the ground state. ${ }^{56}$ This potential should give better glass formation than the harder potentials. For example, it was found that the number of QLV is strongly enhanced compared to the more hard-sphere glasses. ${ }^{18}$ This model glass has been used for the study of the behavior of instantaneous modes, ${ }^{57}$ of the local structure $^{58}$ across the glass transition, and of the influence of the quenching rate on the dynamics. ${ }^{59}$

The advantage of a monatomic model glass is that the dynamics will not depend on parameters such as the size difference between the constituents. One pays for the simplicity by a high instability against crystallization. This limits the observation time at finite temperatures. In binary systems local chemical order hinders long range diffusion. This has already been observed for interstitial atoms in metallic crystals. Self-interstitial atoms are highly mobile which is connected with their low frequency resonant vibrations. These might even be at lower frequencies for impurity interstitials. The related atomic jump motion is, however, locally confined. Diffusion necessitates either an exchange with a host 
atom or an additional different jump with higher activation energy. ${ }^{60}$ The local dynamics is in both cases rather similar but the effect on diffusion differs grossly. One compensation for the instability of our one-atomic model glass is that aging effects are speeded up.

The paper is organized as follows. In the next section we describe the potential and give details of the simulation. In Sec. III we present some basic definitions, discuss the time evolution of the glasses and show how the relaxations are monitored. The properties of these relaxations are discussed in Sec. IV. To round these results off we then consider the short time dynamics at higher temperatures. Finally we discuss our results in the context of previous work and experiments.

\section{COMPUTATIONAL DETAILS}

The soft-sphere glass (SSG) is described by an inverse sixth-power potential

$$
u(r)=\epsilon\left(\frac{\sigma}{r}\right)^{6}+A\left(\frac{r}{\sigma}\right)^{4}+B
$$

To simplify the computer simulation the potential is cut off at $r / \sigma=3.0$, and then shifted by a polynomial, $A(r / \sigma)^{4}+B$, where the choice of $A=2.54 \times 10^{-5} \epsilon$ and $B=-3.43$ $\times 10^{-3} \epsilon$ ensure that the potential and the force are zero at the cutoff. This form of the shifting function was chosen so that its effect is negligible near $r / \sigma=1.0$. Quantities such as the pressure and average potential energy will be changed by a few percent as a result of this truncation, but any changes in the equilibrium structure will be small. At any rate, our interest is in the existence and characterization of relaxations in general, and not in a quantitative description for any specific potential. Without loss of generality one can set the scale factors for energy, length and mass, $\epsilon=\sigma=m=1$. Where we do not explicitly state the units we infer these "system units.",

The inverse sixth-power potential is a well-studied theoretical model that qualitatively mimics many of the structural and thermodynamic properties of bcc forming metals including the existence, in its bcc crystal form, of very soft shear modes. ${ }^{61}$ It gives a high concentration of low-frequency resonant (quasilocalized) modes in the glass. ${ }^{17,18}$ The glasstransition temperature was estimated from the vanishing of the diffusion constant as $k T_{g} \approx 0.085 \epsilon .{ }^{17}$ The melting temperature has been estimated as $k T_{m} \approx 0.19 \epsilon .{ }^{62}$ In this calculation a different cutoff of the potential was used which might shift the value of $T_{m}$ slightly.

We studied systems with 500, 1024, and 5488 particles. They were produced by quenching well equilibrated liquid configurations produced via constant-energy molecular dynamics (MD) simulation with cubic periodic boundary conditions again at a density $\rho \sigma^{3}=1.0$ and temperature $k T / \epsilon$ $\approx 0.54$ (about 2.5 times the melting temperature at this density $\left.^{63}\right)$. All calculations were done at constant volume (density). The average nearest neighbor distance is, independent of temperature, $R_{N N} \approx 1.1 \sigma$. For the simulation, we used the velocity-Verlet algorithm ${ }^{64}$ with a time step of 0.04 in units of $\left(m \sigma^{2} / \epsilon\right)^{1 / 2}$. The liquid is first quenched within the MD simulation by velocity rescaling to a reduced tempera- ture of about $0.005 T_{g}$. To check the numerics some runs were repeated with a reduced time step of 0.004 and also with a modified velocity rescaling frequency. This changed the random times between jumps somewhat but not the relaxations themselves. The quench rate was about $0.015 k /\left(m \sigma^{2} / \epsilon\right)^{1 / 2}$. After the MD quench, each sample was heated to $0.05 T_{g}$ and aged for several 1000 further MD time steps to stabilize the potential energy and to avoid spurious minima. Each system is then quenched to zero temperature using a combination steepest-descent-conjugate-gradient algorithm. ${ }^{65}$ The resulting potential energy equals roughly the one of the fcc crystal of the same density with about $1.5 \%$ Frenkel defects. In all, 60, 21, and 15 different configurations of 500, 1024, and 5488 atoms, respectively, were created in this way and analyzed. Structure factors and radial distribution functions have been shown previously. ${ }^{29}$ To check for the glassy nature of our samples we calculated the two-particle radial distribution functions $g(r)$ and the structure factors $S(k)$. The configurationally averaged values for the smaller samples have been shown in Refs. 17 and 18. For $N=500$ and $N=1024$ we calculated additionally the vibrational spectra. The characteristic changes upon partial crystallization were used to estimate the drop in configurational energy above which crystallization effects might be expected. When we observed it this drop always occurred rapidly.

To study the glasses at elevated temperatures the quenched glasses of 5488 atoms were heated in stages to 5, 10 , and $15 \%$ of the glass temperature. At the two higher temperatures the glasses were observed for 90000 time steps, corresponding to about 5000 vibrations of an average frequency. Taking an average frequency in a simple metal of a few $\mathrm{THz}$ this corresponds to observation times of the order of ns. At $T=0.05 T_{g}$ the glasses were observed for 81000 time steps. At $T=0.05 T_{g}$ some of the glasses were observed for 90000 additional time steps. The smaller samples were observed at four different temperatures between 5 and $12.5 \%$ of $T_{g}$. Due to their greater instability, see next section, they were only observed for 9000 time steps at each temperature.

\section{TIME EVOLUTION AND RELAXATIONS}

\section{A. Definitions}

During the runs the total kinetic and potential energies as well as the total displacement $\Delta R$ from the starting configuration were monitored. We define

$$
\Delta R(t)=\sqrt{\sum_{n}\left[\mathbf{R}^{n}(t)-\mathbf{R}^{n}(0)\right]^{2}}
$$

where $\mathbf{R}^{n}(t)$ is the position vector of particle $n$ at time $t$ and $\mathbf{R}^{n}(0)$ is the one at the starting or reference configuration. $\Delta R(t)$ exhibits both oscillations due to the vibrations and changes due to relaxations, i.e., due to the transition from one local minimum to another. In order to separate the effect of the latter ones, the subject of this paper, it is useful to look at the time averaged displacement

$$
\Delta \overline{R(t)}=\sqrt{\sum_{n}\left\langle\left[\mathbf{R}^{n}(t)-\mathbf{R}^{n}(0)\right]^{2}\right\rangle_{\Delta t}},
$$


where $\langle\cdots\rangle_{\Delta t}$ indicates averaging over a time interval $\Delta t$, typically a few hundred time steps. By quenching the time averaged positions to $T=0$ the configuration of the underlying minima can be found, similar to the procedure of Ref. 66 . At low temperatures where the sample only visits a few minima this procedure is unambiguous. At higher temperatures, let us say $T=0.5 T_{g}$, it depends strongly on the averaging and quench procedure into which minimum the system relaxes. Similar to the above, one can define a $\Delta R^{0}(t)$ for the minimum configurations

$$
\begin{aligned}
\Delta R^{0}(t) & =\sqrt{\sum_{n}\left[\mathbf{R}^{0 n}(t)-\mathbf{R}^{0 n}(0)\right]^{2}} \\
& =\sqrt{\sum_{n}\left(\Delta R^{0 n}\right)^{2}},
\end{aligned}
$$

where $\mathbf{R}^{0 n}(t)$ is now the minimum $(T=0)$ position of particle $n . \Delta R^{0}(t)$ has steps whenever the system moves from one local minimum to another and is constant otherwise. To show the influence of aging on the energy we define $\Delta E^{0}(t)$ in analogy to Eq. (4)

$$
\Delta E^{0}(t)=E^{0}(t)-E^{0}(0),
$$

where $E^{0}(t)$ is the total potential energy of the configuration given by the $\mathbf{R}^{0 n}(t)$.

To classify the relaxations we introduce in analogy to the vibrations a relaxational participation ratio

$$
p_{\Delta R}=\left\{N\left[\sum_{m}\left|\mathbf{R}^{(i) m}-\mathbf{R}^{(f) m}\right|^{4}\right] /\left(\Delta R^{0}\right)^{4}\right\}^{-1},
$$

where $(i)$ and $(f)$ stand for initial and final configuration, respectively, and $\Delta R^{0}$ is given by Eq. (4). For truly local relaxations this participation ratio scales with the system size $N$ as $p_{\Delta R} \propto 1 / N$. We will see that, similar to the findings for the quasi-localized vibrations, this proportionality does not hold exactly. As an alternative to the participation ratio an effective mass

$$
m_{\Delta R}=m\left(\Delta R^{0}\right)^{2} /\left|\Delta \mathbf{R}^{\max }\right|^{2}
$$

can be defined to classify localization. Here $\left|\Delta \mathbf{R}^{\max }\right|$ stands for the maximal atomic displacement. The two definitions of Eqs. (6) and (7) cannot be converted into each other. As a rough guide, one can take that $p_{\Delta R} \approx 0.02$ corresponds to $m_{\Delta R} \approx 20$ for our system and $N=5488$.

\section{B. Time evolution of the glasses}

Figures 1 and 2 (full lines) show the changes of the ensemble averages of the potential energies and displacements of the minima configurations, Eqs. (5) and (4), with time. The average was taken over 14 samples of glasses with $N$ $=5488$. (One sample out of 15 partially crystallized at the highest temperature during the observation time.) The samples were quenched to $T=0$ and then heated to $T$ $=0.004$, aged for a time $\Delta t=3240$, heated to $T=0.008$, aged for $\Delta t=3600$ and once more heated to $T=0.012$ and aged for $\Delta t=3600$. Instead of the final heating stage four of the glasses were kept at $T=0.008$ for an additional $\Delta t=3600$ (dashed line). The dotted lines indicate the heating stages.

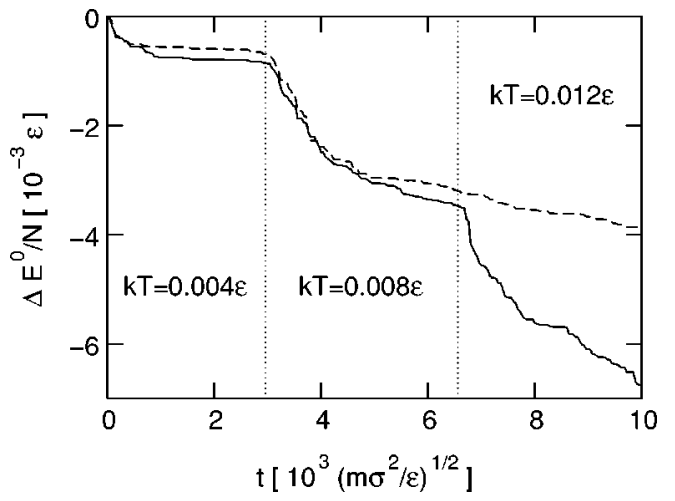

FIG. 1. Change of the configurational part $\Delta E^{0} / N$ of the potential energy per atom during aging and heating (full line) of a glass with $N=5488$ atoms. The different temperature intervals $(k T$ $=0.004,0.008$, and $0.012 \epsilon$ ) are indicated by the dotted lines. The dashed line gives the average over some configurations kept at $k T$ $=0.008 \epsilon$ instead of further heating.

The first 9000 time steps $(\Delta t=360)$ have been omitted from the plots in order to avoid effects of only spuriously stable configurations. One of our configurations showed, toward the end of the last time interval, large relaxations accompanied by a large energy reduction. In order to exclude with certainty a partial crystallization, we excluded this configuration from the two figures.

As expected the potential energy, Fig. 1, drops during aging. After each heating there is a rapid drop with a subsequent leveling off, at least at the lower two temperatures. If one takes the formation energy of a Frenkel defect in the fcc lattice as measure $\left(E^{\mathrm{FP}}=6.05 \epsilon\right)$, the total drop in energy corresponds to the annihilation of only about seven Frenkel defects. The final configurations show no trace of crystallization.

Also the average displacement of the atoms is very small, less than $15 \%$ of the nearest neighbor distance. The largest part of this displacement immediately follows the heating. Striking is the similarity between the energy relaxation, Fig. 1 , and the spatial relaxation, Fig. 2. At the relatively low temperatures considered the corresponding curves using the time averaged quantities, Eq. (3), are very similar to the ones for the underlying ground states.

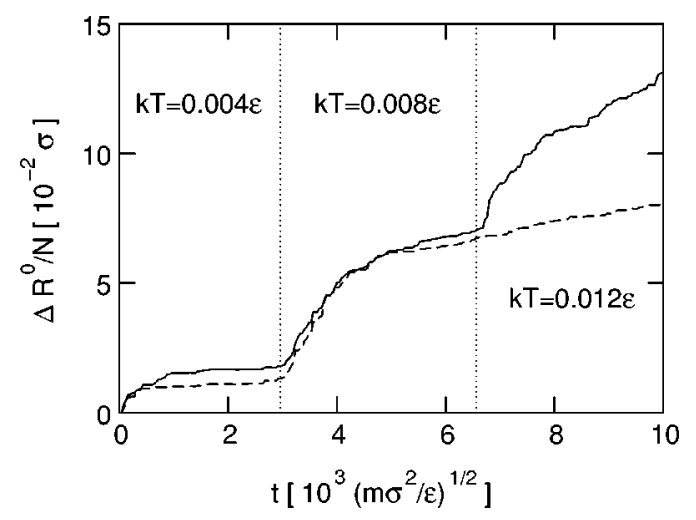

FIG. 2. Average displacement per atom during aging and heating (full line) of a glass with $N=5488$ atoms. The different temperature intervals $(k T=0.004,0.008$, and $0.012 \epsilon)$ are indicated by the dotted lines. The dashed line gives the average over some configurations kept at $k T=0.008 \epsilon$ instead of further heating. 


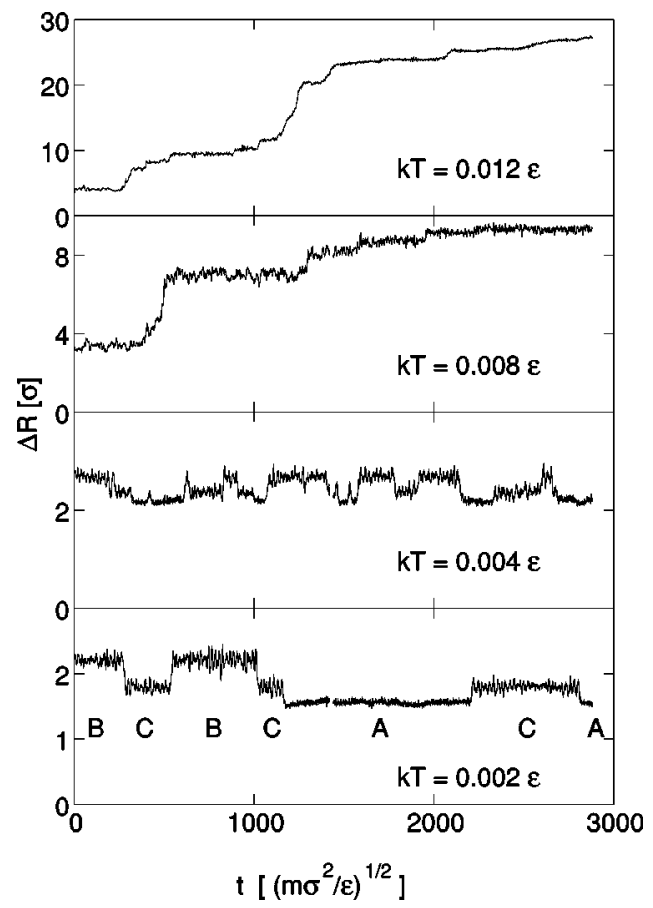

FIG. 3. Displacement relative to a local minimum configuration as function of time for a glass with $N=5488$ at different temperatures. Please note the different scales for $\Delta R$.

In our previous investigation of the vibrational dynamics ${ }^{29}$ we observed for glasses which had run over the first two time segments in Figs. 1 and 2 only a small drop in the number of quasilocal soft modes. The tendency to crystallize depends strongly on the size of the sample used in the MD. Whereas all the samples of $N=5488$ atoms were stable up to temperatures $T=0.008$, three out of 21 samples with $N=1024$ and $30 \%$ of the ones with $N=500$ started to crystallize within one tenth of the time.

\section{Local relaxations}

In the following we want to study single relaxation processes in the glass. Differing from the usual practice we monitor the total displacement according to Eq. (2). This allows the observation of collective jumps of large groups of atoms with total jump lengths of the order of the nearest neighbor distance. In previous studies, e.g., Refs. 32,33, in the supercooled region similar plots for the relaxations of single atoms have been shown. Small distance collective jumps cannot be discerned from the fluctuations in these single atom plots.

Figure 3 shows an example for a glass which had been aged for $\Delta t=5000$ at $k T=0.008 \epsilon$ (corresponding to the dashed line in the third sector of Figs. 1 and 2). The glass was heated during 500 time steps to the respective temperature and subsequently observed for 72000 time steps, i.e., $\Delta t \approx 3000$. This way it was assured that the glass had the same starting configuration at all temperatures. The configuration had been in its lowest energy minimum $A$ immediately before quenching and the shown displacement are relative to this configuration $\mathbf{R}^{n}(t=0)=\mathbf{R}^{A n}(T=0)$ of Eq. (4).

At $k T=0.002 \epsilon$ the glass clearly jumps between three configurations. Let us denote the configurations $A, B, C$. The jump sequence is $B \rightarrow C \rightarrow B \rightarrow C \rightarrow A \rightarrow C \rightarrow A$. By quenching to $T=0 \mathrm{~K}$ we find the potential energy minima shifted relative to each other by $\Delta E^{B}-\Delta E^{A}=1.74 \times 10^{-3} \epsilon$ and $\Delta E^{C}-\Delta E^{A}=1.81 \times 10^{-3} \epsilon$. These energy differences are of the order of the temperature. The corresponding spatial distances between the minimal configurations, Eq. (4), are $\Delta R^{A-B}=1.63 \sigma, \Delta R^{A-C}=0.96 \sigma$, and $\Delta R^{B-C}=1.0 \sigma$, respectively, i.e., they are of the order of the nearest neighbor distance $R_{N N}=1.1 \sigma$. The maximal distance an individual atom travels in these jumps is only $0.3 \sigma$, about a quarter of $R_{N N}$.

The average offset $\overline{\Delta R} \approx 1.5 \sigma$ during the residence time in $A$ corresponds to a vibrational mean square displacement (VMSD) $\left\langle u^{2}(T)\right\rangle_{\mathrm{vib}} \approx 8.2 \times 10^{-4} \sigma^{2}$ per atom. Doubling the temperature the VMSD doubles raising the mean offset to $2.1 \sigma$. The three minima can still be seen but some of the excursions to them are so short-lived that they cannot be distinguished from anharmonic vibrations. Doubling the temperature again the signature of transitions between the minima $A, B$, and $C$ has more or less vanished in the noise but additional, larger relaxations appear. The same story repeats when raising the temperature again by $50 \%$.

From Fig. 3 we have seen that at a given temperature one observes small local relaxations (rearrangements) where groups of atoms move collectively. Raising the temperature these relaxations merge into the vibrational background and new relaxations become observable. In the following we will take a closer and more systematic look at the properties.

\section{PROPERTIES OF SINGLE RELAXATIONS}

\section{A. Procedure}

For a systematic study of the single relaxations which the glasses undergo during their history, Figs. 1 and 2, we have to analyze their $\Delta R(t)$ as shown in Fig. 3, for example. We calculate average displacements according to Eq. (3) averaging over 900 time steps which corresponds to somewhat over three periods of the quasilocalized vibrations. For $\mathbf{R}^{n}(0)$ we take the positions in the starting minimum. If $\overline{\Delta R}$ exceeds a value of $0.2 \sigma$ the new averaged configuration is considered a possible minimum. The possible candidates for new configurations are then quenched to $T=0$ to see whether they really belong to a new local minimum of the potential energy. Configurations which thus relax to their predecessor are discarded. Using this procedure the three different minima are obviously recorded at $T=0.002 \epsilon / k$ but not for the shortest excursions at $T=0.012 \epsilon / k$. At $T=0.008 \epsilon / k$ the small maximum around $t=140$ is caused by an underlying local minimum of the potential energy. The large jump around $t$ $=550$, on the other hand, is not resolved into smaller subjumps, the possible intermediate steps seen as tiny spikes are neglected. For the smaller sizes $(N=500$ and $N=1024)$ the relaxations were taken at temperatures ranging between $0.05 T_{g}$ and $0.125 T_{g}$, whereas for $N=5488$ the shown relaxations were observed during the aging shown in Figs. 1 and 2.

\section{B. Structure of relaxations}

We observed on average in each of the 15 samples $(N$ $=5488) 6,15$, and 15 relaxations at the temperatures $T / T_{g}$ 


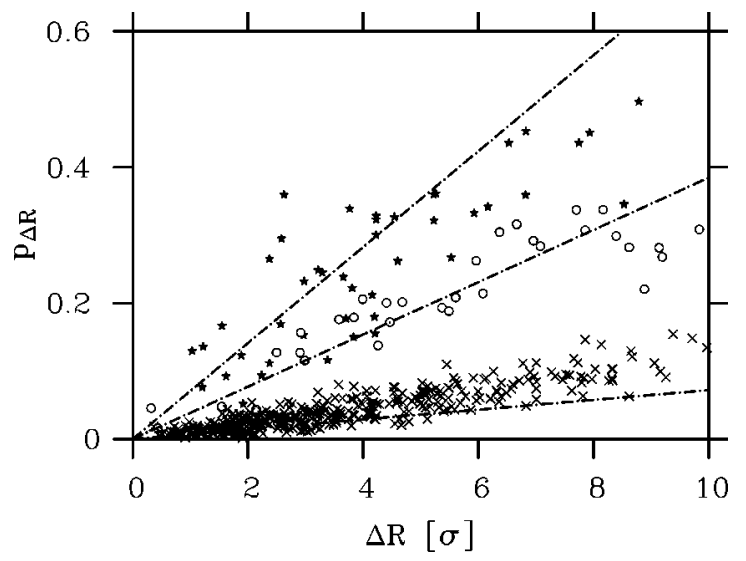

FIG. 4. Participation ratio of relaxations against jump distance for configurations with $N=500(\star), N=1024(\bigcirc)$, and $N=5488$ ( $\times$ ) at temperatures up to $T \approx 0.15 T_{g}$. The lines are guides to the eye which give the ideal scaling of the participation ratio with system size.

$=0.05,0.1$, and 0.15 respectively. In Fig. 4 the participation ratios of the observed jumps are plotted against the jump length, independent of the observation temperature. Shown are the results for the three system sizes. The lines are drawn as guides to the eye to show the approximate scaling of the participation ratio with the system size.

Looking, particularly, at the larger jump distances one sees that the scaling of the participation ratio with system size does not strictly hold. This resembles the similar behavior observed for the quasilocalized vibrations. ${ }^{29}$ With increasing system size the probability to find more than one relaxation center in the system increases. Under suitable conditions the relaxations will interact, which in turn increases their participation ratio. Since the interaction strength increases with the total displacement long distance jumps will interact more strongly than short distance ones. Depending on the energetics different scenarios can evolve. Interaction can lead to a growth of single relaxations with temperature if two relaxations attach to each other and the activation energy of one of the two constituents is lower than both the ones of the partner and the pair. This situation was illustrated in Ref. 40. Another possibility is that the pair is most favored. This is the case for the low-temperature relaxations of Fig. 3. There, configuration $B$ is obtained from $A$ by flipping a pair of relaxators, whereas only one of the two relaxators is flipped in the transition $A \rightarrow C$. An isolated flipping of the second relaxator needs higher energies. It causes the little blip in $\Delta R$ for $k T=0.008 \epsilon$ at $t \approx 150$. Different from our work on the vibrations we have not tried to systematically separate the observed relaxations into their local constituents.

Taking only the jumps with energy gain less than $k T$, the average jump length increases in this temperature range from $\Delta R \approx R_{N N} \approx 1.1 \sigma$ to $\Delta R \approx 1.5 R_{N N}$. The maximum distance a single atom moves in these jumps increases from about $0.2 R_{N N}$ to $0.3 R_{N N}$. The average participation ratio grows from approximately 0.015 to 0.021 . These numbers correspond to localizations to about 18 to 22 atoms. The increase in the total jump length $\Delta R$ with temperature is a combined effect of longer jumps of the single atoms and an increase in the number of atoms participating. The strongest localization

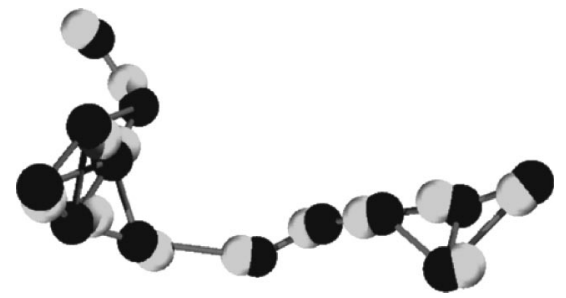

FIG. 5. Relaxation at $T \approx 0.1 T_{g}$ in a glass with $N=5488(\Delta R$ $\left.=1.98 \sigma, p_{\Delta R}=0.018\right)$. Shown are all atoms whose squared displacement is more than $30 \%$ of the maximal one, full circles: initial position, shaded circles: final position.

we observed is to about six atoms, which is still more than the typical value found by Heuer and Silbey. ${ }^{31}$ We cannot say for certain whether this is due to the different interaction model or to the finite temperature in our simulation. If we do not restrict the averaging to small energy gains the average values for $\Delta R$ and $p_{\Delta R}$ approximately double. This might be due to a larger number of multiple jumps thus included.

As already observed earlier ${ }^{40}$ the relaxations are collective motions along closely packed directions which gives them chainlike structures. This structure is more pronounced at higher temperatures. As an illustration Fig. 5 shows the most active atoms of a relaxation at $T \approx 0.1 T_{g}$. The total jump length is $\Delta R=1.98 \sigma$, the participation ratio $p_{\Delta R}=0.018$, and the effective mass $m_{\Delta R}=23$. Shown are all atoms whose squared displacement is more than $30 \%$ of the maximal one. The displacements of the individual atoms are shown true to scale by the shaded spheres.

To classify the structure more quantitatively we define, again following Ref. 29 a dimensionality of the relaxations and calculate for each relaxation $j$ the tensor

$$
G_{\alpha \beta}(j)=\frac{\sum_{n}\left|\Delta \mathbf{R}^{n}(j)\right|^{\mu}\left(R_{\alpha}^{n}-R_{\alpha}^{\text {c.m. }}\right)\left(R_{\beta}^{n}-R_{\beta}^{\text {c.m. }}\right)}{\sum_{n}\left|\Delta \mathbf{R}^{n}(j)\right|^{\mu}},
$$

where we take the exponents $\mu=2$ and $\mu=4$, corresponding to the effective mass and participation ratio, respectively. $\mathbf{R}^{\text {c.m. }}$ is the corresponding center of mass coordinate of the relaxation:

$$
\mathbf{R}^{\text {c.m. }}=\frac{\sum_{n}\left|\Delta \mathbf{R}^{n}(j)\right|^{\mu} \mathbf{R}^{n}}{\sum_{n}\left|\Delta \mathbf{R}^{n}(j)\right|^{\mu}} .
$$

Diagonalizing $G$ we obtain three eigenvalues $\rho^{i}(j, \mu)$ and from these an average gyration radius ${ }^{20,67}$

$$
R_{\mathrm{gyr}}(j, \mu)=\sqrt{\frac{1}{3} \sum_{i} \rho^{i}(j, \mu)} .
$$

If a relaxation is localized on a single atom $R_{\mathrm{gyr}}=0$. For an extended relaxation it is the root-mean square distance with the weight determined by $\mu$. An effective dimension of the relaxation can be defined as 


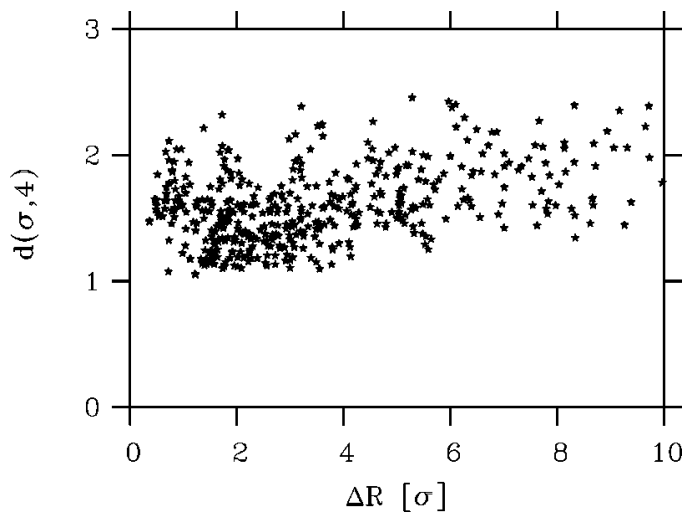

FIG. 6. Dimension of jumps related to participation ratio in a glass with $N=5488$ at temperatures up to $T \approx 0.15 T_{g}$

$$
d(j, \mu)=\sum_{i} \rho^{i}(j, \mu) / \max \rho^{i}(j, \mu) .
$$

Averaging over all relaxations in the systems with $N$ $=5488$ we get weighting with the effective mass a gyration radius $\bar{R}_{\text {gyr }}(2)=5.8 \sigma$ and a dimension $\bar{d}(2)=2.1$. Taking the participation ratio as weight the values are slightly reduced to $\bar{R}_{\mathrm{gyr}}(4)=4.1 \sigma$ and $\bar{d}(4)=1.6$. These values are rather similar to the ones found for the quasilocalized vibrations in the same system. ${ }^{29}$ If we take the average only over relaxations with $\Delta R<2 \sigma$ the gyration radii are somewhat reduced to $\bar{R}_{\text {gyr }}(2)=4.4 \sigma$ and $\bar{R}_{\text {gyr }}(4)=2.2 \sigma$ whereas the dimensions stay nearly the same. The effective mass related values are always higher than the ones related to the participation ratio reflecting the different weight of the long range displacements.

Figure 6 summarizes the results for $\bar{d}(4)$. We find a considerable spread of values. This is related to bends and side branching of the relaxational chain structures. On the other hand, the dimensionality is nearly independent of the jump length $\Delta R$, see Fig. 6 . The slight increase with $\Delta R$ could be due to unresolved multiple jumps.

\section{Correlations}

We have seen that in an individual relaxation chains of atoms jump collectively. These jumps are not uncorrelated events but successive jumps tend to involve the same atoms. As a quantitative measure we introduce the correlation

$$
c_{\Delta R}\left[j, j^{\prime}\right]=\frac{1}{\Delta R[j] \Delta R\left[j^{\prime}\right]} \sum_{n} \Delta R^{n}[j] \cdot \Delta R^{n}\left[j^{\prime}\right],
$$

where $j$ and $j^{\prime}$ denote successive relaxations. As opposed to a definition in terms of the product of the jump vectors $\Delta \mathbf{R}^{n}[j]$, successive jumps centered on the same atoms contribute also if they are orthogonal to each other. Direct return jumps give $c_{\Delta R}\left[j, j^{\prime}\right]=1$. The correlation between jumps $A$ $\rightarrow B$ and $B \rightarrow C$, of Fig. 3, is $c_{\Delta R}[A \rightarrow B, B \rightarrow C]=0.87$. For completely uncorrelated jumps one would get values of the order of $1 / N$.

Figure 7 (bottom) shows the observed distribution of the correlation values for the $N=5488$ system averaged over all relaxations observed either at the lowest temperature $T$

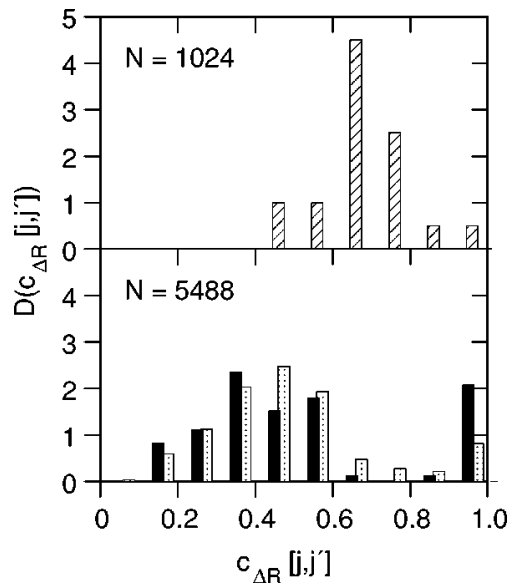

FIG. 7. Bottom: Distribution of correlations between successive jumps for $N=5488$ at $T \approx 0.05 T_{g}$ (solid bar) and for all temperatures up to $T \approx 0.15 T_{g}$ (dotted bar). Top: Distribution of correlations between successive jumps for $N=1024$ at temperatures up to $T$ $\approx 0.125 T_{g}$.

$\approx 0.05 T_{g}$ (solid bars) or during the whole time range of Fig. 1 (dotted bars). It is evident that all observed jumps are correlated with their immediate neighbors in time. We observe two maxima in the distributions, around 0.4 and at 1 . The latter value stems from immediate return jumps $(A \rightarrow B$ $\rightarrow A$ ). At the lowest temperature this accounts for $20 \%$ of the observed jumps. Raising the temperature, this value drops rapidly, reflecting the greater freedom of the material to cross barriers between potential minima. The broad distribution around $c_{\Delta R}\left[j, j^{\prime}\right] \approx 0.4$ originates from both: jumps which cannot be reversed due to a too strong drop in potential energy and reversible jumps involving more than two minima.

The observed correlation values depend nontrivially on the system size. This is exemplified by the upper part of Fig. 7 where the corresponding distribution for the smaller samples with $N=1024$ is shown. Striking is the shift of the main peak to higher correlations for the smaller system size. With increasing size more and more only weakly coupled relaxations in distant regions become possible. In the limit $N \rightarrow \infty$ the correlation between successive jumps will decrease to zero since there will always be a multitude of distant jumps intervening. In fact already with our small samples we frequently observe higher correlations to later jumps than to the next one.

The correlation between jumps holds at the investigated temperatures over considerable times, i.e., the relaxations remain centered on the same "active groups" of atoms. This is illustrated in Fig. 8 which shows the initial positions of all atoms which have moved by more than $0.35 \sigma$ during a time interval $\Delta t=3600\left(m \sigma^{2} / \epsilon\right)^{1 / 2}$ at $T \approx 0.15 T_{g}$. The total displacement is $\Delta R=8 \sigma$, the participation ratio $p_{\Delta R}=0.077$, and the effective mass $m_{\Delta R}=107$ still quite small compared to the total mass 5488. Compared to the single relaxation in Fig. 5 this combined one is quite extended. One can envisage it as being built up of some more chainlike constituents. More or less independent of the large relaxation there is an additional strongly localized one.

Another correlation is postulated by the soft potential model $^{11,12}$ where local relaxations are related to soft local vibrations. We have seen that relaxations interact and the 


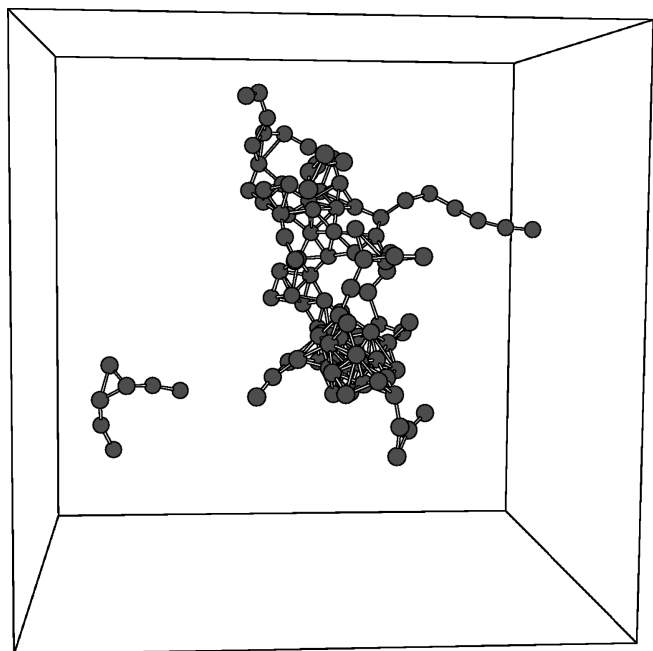

FIG. 8. Initial positions of atoms displaced by more than $0.35 \sigma$ in a time interval $\Delta t=3600\left(m \sigma^{2} / \epsilon\right)^{1 / 2}$ at $T \approx 0.15 T_{g}$. Total displacement $\Delta R=8.02 \sigma, p_{\Delta R}=0.077$.

same holds for the soft vibrations. ${ }^{29}$ These interactions are, however, not included in the SPM. In Ref. 29 we extracted the "noninteracting"' soft modes and showed that the SPM is applicable to these. To do the same procedure for the relaxations seems rather tedious. Instead we restrict ourselves to the samples with $N=500$ where due to the size and hence the lack of partners this interaction is strongly reduced for both vibrations and relaxations. We calculate the projection of the normalized jump vector onto the eigenvectors $\mathbf{e}_{\text {soft }}^{n}$ of the soft vibrations

$$
P=\frac{1}{\Delta R} \sum_{\text {soft }} \sum_{n}\left(\Delta \mathbf{R}^{n} \mathbf{e}_{\mathrm{soft}}^{n}\right)
$$

where we use in each glass between 1 and 3 soft modes as determined in Refs. 17 and 18. $P$ should vary between 1 for total correlation and $1 / N$ if jumps and soft vibrations are unrelated. Figure 9 shows that the truth lies in between. There is a strong correlation but the value of $P=1$ assumed in the SPM is only a first approximation. In Ref. 41 we have shown for amorphous Se that $P$ approaches its limit quite fast if one increases the subspace of soft modes. We expect

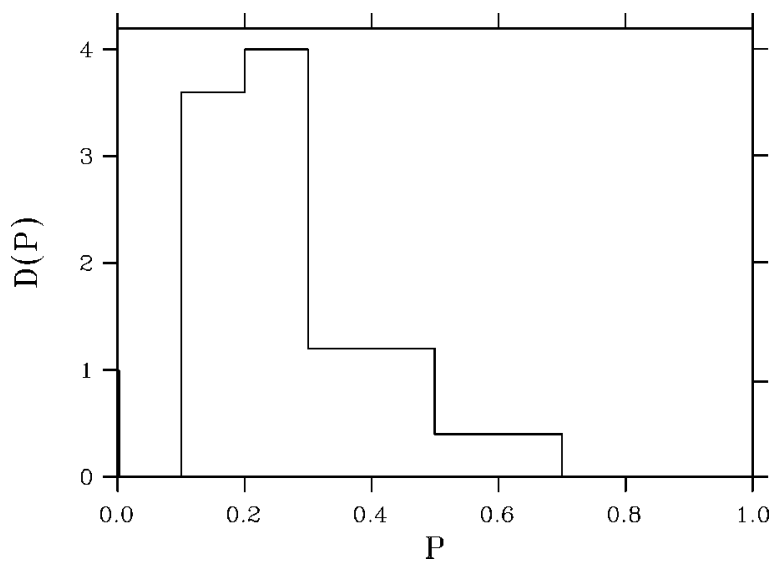

FIG. 9. Distribution of the projections of the normalized jump vectors onto the eigenvectors of the quasilocalized soft vibrations for $N=500$. the same for our soft sphere glass. The highest values of $P$ are obtained for the most local relaxations which do not lead to a large energy change.

\section{HIGHER TEMPERATURES}

Due to the tendency to crystallize the SSG can at higher temperatures only be observed on short time scales. It is, however, possible to monitor the glass for short times after a rapid heating to a given temperature. As throughout the paper the sample volume is kept constant. Compared to constant pressure conditions, this means for the soft-sphere potential, a distortion of the temperature axis. We define the average mean square displacement (MSQD) as usual:

$$
\left\langle u^{2}(\Delta t)\right\rangle=\left\langle\sum_{n}\left|\mathbf{R}^{n}\left(t_{0}+\Delta t\right)-\mathbf{R}^{n}\left(t_{0}\right)\right|^{2}\right\rangle .
$$

$\left\langle u^{2}\right\rangle$ is made up from two approximately independent contributions from the vibrations and relaxations or jump processes, respectively:

$$
\left\langle u^{2}\right\rangle=\left\langle u_{\mathrm{vib}}^{2}\right\rangle+\left\langle u_{\mathrm{rel}}^{2}\right\rangle .
$$

In classical harmonic approximation and for averaging times larger than the typical vibration period

$$
\left\langle u_{\mathrm{vib}}^{2}\right\rangle=\left\langle\frac{3 k T}{m} \int d \omega \frac{1}{\omega^{2}} g^{n}(\omega)\right\rangle,
$$

where $g^{n}(\omega)$ is the local vibrational spectrum of atom $n$. $\left\langle u_{\mathrm{vib}}^{2}\right\rangle$ increases linearly with temperature and is independent of the time difference $\Delta t$, except for $\Delta t$ values smaller than the typical vibration period. In the liquid state and for sufficiently long observation times one has for the second part

$$
\left\langle u_{\mathrm{rel}}^{2}\right\rangle \sim 6 D(T) \Delta t
$$

At the highest temperatures the diffusion constant increases linearly with temperature in accordance with simulations on liquid $\mathrm{Rb}^{68}$ In the solid and in the under-cooled melt the behavior will be much more complex. We do not reach the asymptotic time regime, and the diffusion constant will have an exponential or stretched exponential dependence on temperature. With increasing temperature the number of relaxation processes will increase and at the same time the displacement in an individual relaxation will also increase. We, therefore, still expect an increase of $\left\langle u_{\text {rel }}^{2}\right\rangle$ with temperature as well as with averaging time. The latter will, however, not be linear since during the observation time some of the relaxations will be reversed which would lead ultimately to a time independent contribution similar to the vibrational one. Counterbalancing this effect somewhat is the triggering of relaxations by relaxations which would be observed more likely in the longer times. These effects can be clearly observed in Fig. 10.

We used the glasses with $N=5488$ after aging to the end of the second temperature interval $T \approx 0.1 T_{g}$ of Fig. 1 as starting configurations. These were then rapidly heated to the observation temperature and after a short equilibration lasting 200 time steps observed during $\Delta t=10$ (broken line) and $\Delta t=40$ (solid line), respectively. If one fixes the maximal 


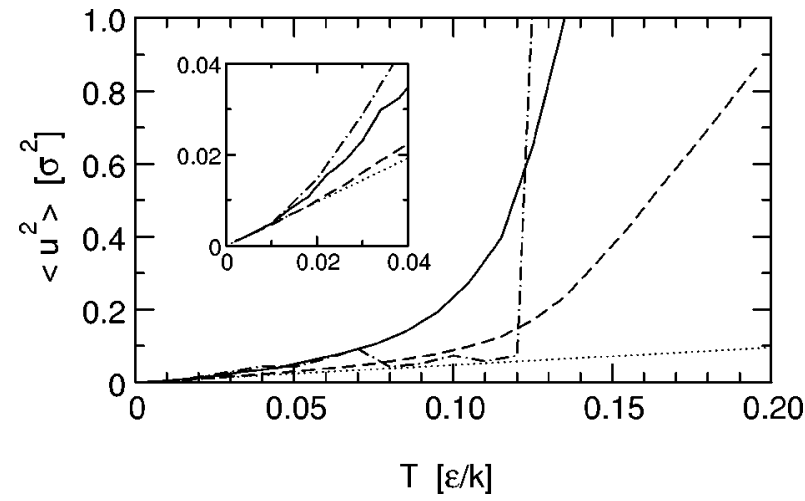

FIG. 10. Mean square displacement after $\Delta t=40$ (solid line) and $\Delta t=10$ (dashed line). The dotted line shows the extrapolated vibrational contribution. The dash dotted line shows the mean square displacement after $\Delta t=80$ after the material was aged at the given temperature for $\Delta t=800$. The density was kept constant at $\rho \sigma^{3}=1$.

frequency around $5 \mathrm{THz}$, typical for a soft metal, this corresponds to a few ps, i.e., observation times of neutron scattering experiments. At the lowest temperatures, $k T<0.01 \epsilon$ the total MSQD $\left\langle u^{2}\right\rangle$ is nearly entirely vibrational. The extrapolation to higher temperatures is indicated by the dotted line. Above $k T \approx 0.12 \epsilon$ the MSQD exhibits liquid behavior. The intermediate temperature range comprises the glass as well as the undercooled liquid which we cannot distinguish from this measurement. Apart from the lowest temperatures, where the statistics is very poor, the factor between the relaxational contributions for the two time intervals $\Delta t$ varies between 4 and 5 .

To see the effects of the inherent tendency to crystallization we also calculated the MSQD averaging for $\Delta t=80$ after aging the samples for $\Delta t_{\text {aging }}=800$ at each temperature. The result is shown by the dash-dotted line in Fig. 10. For $k T<0.04 \epsilon$ no dramatic effects occur. At $k T=0.05$ the MSQD drops to the value obtained for half the observation time and finally above $k T=0.07$ to the vibrational contribution. This clearly shows crystallization, albeit to rather defective structures whose energies correspond to Frenkel-pair concentrations of about $1 \%$. The little maximum around $k T$ $=0.1$ is then the trace of some defect annihilation. The sudden increase at $k T=0.12$ shows the melting of the samples. At the higher temperatures the curve follows the behavior of the other two cases.

An important feature characterizing the dynamics in glasses is the deviation from Gaussian behavior (nonGaussianity). Following Raman ${ }^{69}$ we introduce the nonGaussianity parameter $\alpha_{n}$ as the normalized ratio of the moments of the displacements. For the lowest moments this gives

$$
\alpha_{2}(\Delta t)=\frac{3\left\langle u^{4}\right\rangle(\Delta t)}{5\left[\left\langle u^{2}\right\rangle(\Delta t)\right]^{2}}-1 .
$$

The solid and dashed lines in Fig. 11 show the temperature variation of $\alpha_{2}(\Delta t)$ for two time intervals $\Delta t=60$ and $\Delta t$ $=15$, respectively. For the short time interval the value stays fairly constant around 0.3 before it drops to zero above the melting temperature. For the longer time interval $\alpha_{2}(\Delta t)$

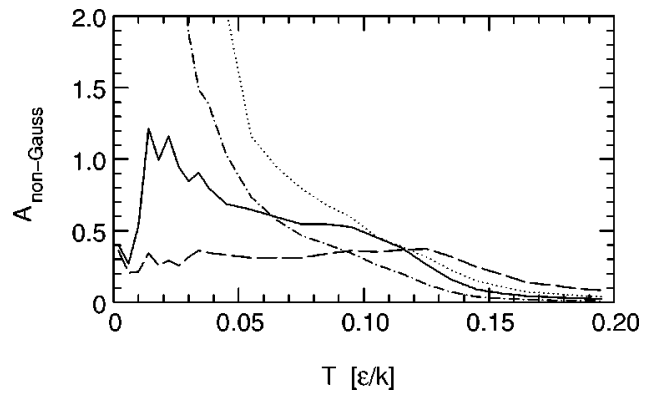

FIG. 11. Non-Gaussianity parameter for $\Delta t=60$ (solid line) and $\Delta t=15$ (dashed line). The non-Gaussianity parameters of the relaxational contributions alone are shown by the dash-dotted and dotted lines. The density was kept constant at $\rho \sigma^{3}=1$.

grows in the amorphous state to much higher values around 1 , drops to about 0.5 and then drops to zero faster than the short time value. This reflects the different weighting of the vibrational and relaxational contributions. At finite temperatures the latter always dominates for long enough times. The maximum of $\alpha_{2}(\Delta t)$ around $k T=0.03 \epsilon$ indicates that during $\Delta t$ considerable relaxations have taken place which, however, involve only a limited number of atoms, as was illustrated in Fig. 8.

Analogous to Eq. (15) the fourth moments can be decomposed into vibrational and relaxational parts

$$
\left\langle u^{4}\right\rangle=\left\langle u_{\mathrm{vib}}^{4}\right\rangle+\left\langle u_{\mathrm{rel}}^{4}\right\rangle+\frac{10}{3}\left\langle u_{\mathrm{vib}}^{2}\right\rangle\left\langle u_{\mathrm{rel}}^{2}\right\rangle .
$$

The total non-Gaussianity can then be separated into a vibrational and a relaxational part ${ }^{70}$

$$
\alpha_{2}(\Delta t)=\frac{\alpha_{2}^{\mathrm{vib}}(\Delta t)\left[\left\langle u_{\mathrm{vib}}^{2}\right\rangle\right]^{2}+\alpha_{2}^{\mathrm{rel}}(\Delta t)\left[\left\langle u_{\mathrm{rel}}^{2}\right\rangle\right]^{2}}{\left[\left\langle u_{\mathrm{vib}}^{2}\right\rangle+\left\langle u_{\mathrm{rel}}^{2}\right\rangle\right]^{2}} .
$$

Neglecting anharmonicity we can extrapolate the moments of the vibrational displacements from low temperatures and using Eqs. (15), (19), and (20) calculate the non-Gaussianity of the relaxations alone. The results for the two values of $\Delta t$ are shown by the dash-dotted and dotted lines in Fig. 11. As to be expected the non-Gaussianity $\alpha_{2}^{\text {rel }}(\Delta t)$ of the relaxations is larger for the smaller time interval and increases rapidly upon lowering the temperature below the glass transition.

Due to the short lifetime of the soft sphere glass at finite temperatures these short time effects cannot directly be observed in real experiments. Our computer simulation compresses the effects observed in real materials into short time spans. The qualitative behavior corresponds to the one observed in real materials. ${ }^{70}$

\section{DISCUSSION AND CONCLUSION}

In the present paper we have studied relaxations in a monatomic soft-sphere glass. Using sufficiently large samples in the simulation the glasses can be kept at temperatures up to $15 \%$ of the glass transition temperature in a metastable state for several thousand vibrational periods. At higher temperatures the stability is drastically reduced and the glass can only be observed for short times after rapid heating to the 
desired temperature. This disadvantage is somewhat compensated by the concomitant rapid aging which is a notorious problem in the more stable binary glasses. At low temperatures we can identify a number of local jump processes. These can be envisaged as collective motions of chainlike structures of ten and more atoms where a single atom moves only a fraction of a nearest neighbor distance. We observe a number of reversible jumps, i.e., jumps connecting a limited number of configurations belonging to local energy minima of similar value. The majority of jumps, especially after heating, leads to minima of lower energy, corresponding to aging. No qualitative difference between the jumps was observed. This is in accordance with the assumptions of the soft potential model and the results of Heuer and Silbey using different simulation techniques. ${ }^{31,71}$ Our earlier findings ${ }^{40}$ are confirmed by the more detailed study.

Increasing the temperature the total jump length, as well as the number of strongly participating atoms, grows. A similar increase has also been observed in simulations of $\mathrm{Se}^{72,73}$ In the instantaneous mode spectrum it is observed as an increase of the participation ratio of the modes with imaginary frequency. ${ }^{57}$

The observed jumps are correlated with the quasilocal vibrations discussed earlier. ${ }^{17,29}$ The correlation is, however, weaker than postulated in the soft potential model. At the lowest temperatures the number of atoms participating in the jumps is clearly smaller than one would expect from the corresponding vibrations. The growth of the participation ratio with temperature is clearly outside the scope of the soft potential model in its present form and the model should be extended to account for these effects.

If one makes the plausible assumption that the observed relaxations are not only local events but are also the elementary step in diffusion, our results clearly indicate in this oneatomic glass a collective mechanism as opposed to a vacancy one. In multicomponent glasses we would expect to find this mechanism for the majority, structure forming component but not necessarily for the other atoms. We find low dimensional chainlike structures for the jumping atoms. This is in disagreement with the assumption of collectivity due to holes. ${ }^{46}$ Whereas the total displacement of such a chain is of the order of the atomic distance the single atoms move only a fraction of this distance. We observed neither a qualitative difference between reversible jumps and irreversible ones, causing a marked reduction in energy, nor do we observe any single atom jumps.
Not only does collectivity hold for a single jump process, but there is also a marked correlation between subsequent jump events. This collectivity seems to hold well into the liquid state. ${ }^{35-37}$ The "collective regions" do not form compact structures whence it is difficult to associate them with "frozen in unrelaxed regions" as done, e.g., in Ref. 43. Such regions had been neither seen in the vibrational structures studied in Ref. 29.

The quasilocalized low frequency vibrations are centered at atoms in a local environment distinct from the average one. ${ }^{17}$ The shell of nearest neighbors is compressed but less dense. The deficiency is made up at distances between the first and second shell. Due to the correlation this holds also for the local relaxations (jumps). If one wants to talk of defects this corresponds more to the signature of an interstitialcy than a vacancy. Speaking of free volume one should keep in mind that excess volumes due to a vacancy or a self-interstitial in metals are similar. ${ }^{60}$ The difference is merely in its localization. A reduction of volume upon aging does not give clear information on the nature of the annealed "defects." We have reported earlier ${ }^{29}$ a small reduction of soft centers upon aging.

The results of the computer simulation agree well with experimental results. Low-temperature thermal relaxations in glasses are observed, e.g., by internal friction and neutron scattering experiments. Measuring the isotope effect it has been shown that diffusion in metallic glasses is via collective motion involving numbers of particles similar to our results. $^{50,54}$

In the liquid state, upon cooling towards the glass transition, the distribution of the atomic mean square displacements deviates increasingly from a Gaussian distribution, in agreement with earlier simulations. ${ }^{74}$ The "rapid quench values" for the glassy state show for sufficiently long observation times a further increase upon cooling. This is due to a strong non-Gaussianity of the relaxations. For low temperatures this contribution eventually diminishes due to the rare occurrence of relaxations. The non-Gaussianity drops to the value given by the vibrations.

\section{ACKNOWLEDGMENTS}

We are grateful for many discussions to U. Buchenau, A. Heuer, and R. Zorn. This work was partially supported by the Deutsche Forschungsgemeinschaft in the Schwerpunkt "Unterkühlte Metallschmelzen: Phasenselektion und Glasbildung."
*Present address: Institut für Physikalische und Theoretische Chemie, Universität Bonn, D-53115 Bonn, Germany.

${ }^{1}$ Amorphous Solids: Low Temperature Properties, edited by W. A. Phillips (Springer-Verlag, Berlin, 1981).

${ }^{2}$ Glassy Metals I, edited by H.-J. Günterodt and H. Beck (Springer-Verlag, Berlin, 1981).

${ }^{3}$ W. Phillips, J. Low Temp. Phys. 7, 351 (1972).

${ }^{4}$ P. W. Anderson, B. I. Halperin, and C. M. Varma, Philos. Mag. 25, 1 (1972).

${ }^{5}$ W. W. Scott and R. K. McCrone, Phys. Rev. B 1, 3515 (1970)

${ }^{6}$ K. S. Gilroy and W. A. Phillips, Philos. Mag. 43, 735 (1981).

${ }^{7}$ S. Hunklinger and A. K. Raychaudhuri, in Progress in Low Tem- perature Physics IX, edited by D. F. Brewer (Elsevier, Amsterdam, 1986).

${ }^{8}$ U. Buchenau, H. M. Zhow, N. Nücker, K. S. Gilroy, and W. A. Phillips, Phys. Rev. Lett. 60, 1318 (1988).

${ }^{9}$ R. Keil, G. Kasper, and S. Hunklinger, J. Non-Cryst. Solids 164166, 1183 (1993).

${ }^{10}$ K. A. Topp and D. G. Cahill, Z. Phys. B 101, 235 (1996).

${ }^{11}$ V. G. Karpov, M. I. Klinger, and F. N. Ignatiev, Sov. Phys. JETP 57, 439 (1983).

${ }^{12}$ M. A. Il'in, V. G. Karpov, and D. A. Parshin, Sov. Phys. JETP 65, 165 (1983).

${ }^{13}$ U. Buchenau, Y. M. Galperin, V. L. Gurevich, and H. R. Schober, Phys. Rev. B 43, 5039 (1991). 
${ }^{14}$ U. Buchenau, Yu. M. Galperin, V. L. Gurevich, D. A. Parshin, M. A. Ramos, and H. R. Schober, Phys. Rev. B 46, 2798 (1992).

${ }^{15}$ L. Gil, M. A. Ramos, A. Bringer, and U. Buchenau, Phys. Rev. Lett. 70, 182 (1993).

${ }^{16}$ A. P. Sokolov, R. Calemczuk, B. Salce, A. Kisliwk, D. Quitmann, and E. Duval, Phys. Rev. Lett. 78, 2405 (1997).

${ }^{17}$ B. B. Laird and H. R. Schober, Phys. Rev. Lett. 66, 636 (1991).

${ }^{18}$ H. R. Schober and B. B. Laird, Phys. Rev. B 44, 6746 (1991).

${ }^{19}$ S-P. Chen, T. Egami, and V. Vitek, Phys. Rev. B 37, 2440 (1988).

${ }^{20}$ W. Jin, P. Vashishta, R. K. Kalia, and J. P. Rino, Phys. Rev. B 48, 9359 (1993).

${ }^{21}$ C. Oligschleger and H. R. Schober, Physica A 201, 391 (1993).

${ }^{22}$ J. Hafner and M. Krajci, J. Phys.: Condens. Matter 6, 4631 (1994).

${ }^{23}$ P. Ballone and S. Rubini, Phys. Rev. B 51, 14962 (1995).

${ }^{24}$ L. D. V. Ee, B. J. Thijsse, and J. Sietsma, J. Non-Cryst. Solids 205-207, 641 (1996).

${ }^{25}$ M. Cho, G. R. Fleming, S. Saito, I. Ohmine, and R. M. Stratt, J. Chem. Phys. 100, 6672 (1994).

${ }^{26}$ J. Hafner and M. Krajci, J. Phys.: Condens. Matter 5, 2489 (1993)

${ }^{27}$ R. Biswas, A. M. Bouchard, W. A. Kamitakahara, G. S. Grest, and C. M. Soukoulis, Phys. Rev. Lett. 60, 2280 (1988).

${ }^{28}$ J. Fabian and P. B. Allen, Phys. Rev. Lett. 77, 3839 (1996).

${ }^{29}$ H. R. Schober and C. Oligschleger, Phys. Rev. B 53, 11469 (1996).

${ }^{30}$ J. Fabian and P. B. Allen, Phys. Rev. Lett. 79, 1885 (1997).

${ }^{31}$ A. Heuer and R. J. Silbey, Phys. Rev. Lett. 70, 3911 (1993).

${ }^{32}$ H. Miyagawa, Y. Hiwatari, B. Bernu, and J. Hansen, J. Chem. Phys. 88, 3879 (1988).

${ }^{33}$ G. Wahnström, Phys. Rev. A 44, 3752 (1991).

${ }^{34}$ S. Sanyal and A. K. Sood, Europhys. Lett. 34, 361 (1996).

${ }^{35}$ H. Teichler, Defect Diffus. Forum 143-147, 717 (1997).

${ }^{36}$ T. Schuler, U. Hamlescher, P. Scharwaechter, and W. Frank, Defect Diffus. Forum 143-147, 753 (1997).

${ }^{37}$ H. R. Schober, C. Gaukel, and C. Oligschleger, Defect Diffus. Forum 143-147, 723 (1997).

${ }^{38}$ C. Donati, J. F. Douglas, W. Kob, S. J. Plimpton, P. H. Poole, and S. C. Glotzev, Phys. Rev. Lett. 80, 2338 (1998).

${ }^{39}$ C. Gaukel and H. R. Schober, Solid State Commun. 107, 1 (1998)

${ }^{40}$ H. R. Schober, C. Oligschleger, and B. B. Laird, J. Non-Cryst. Solids 156, 965 (1993).

${ }^{41}$ C. Oligschleger and H. R. Schober, Solid State Commun. 93, 1031 (1995).

${ }^{42}$ Y. Limoge, Acta Metall. Mater. 38, 1733 (1990).

${ }^{43}$ W. Frank, U. Hamlescher, K. Kronmüller, R. Scharwaechter, and T. Schuler, Phys. Scr. T66, 201 (1996).

${ }^{44}$ W. Frank, Defect Diffus. Forum 143-147, 695 (1997).
${ }^{45}$ M. H. Cohen and G. S. Grest, Phys. Rev. B 20, 1077 (1979).

${ }^{46}$ J. Sietsma and B. J. Thijsse, Phys. Rev. B 52, 3248 (1995).

${ }^{47}$ A. Grandjean, P. Blanchard, and Y. Limoge, Phys. Rev. Lett. 78, 697 (1997).

${ }^{48}$ H. J. Höfler, R. S. Averback, G. Rummel, and H. Mehrer, Philos. Mag. Lett. 66, 301 (1992).

${ }^{49}$ P. Klugkist, K. Rätzke, S. Rehders, P. Troche, and F. Faupel, Phys. Rev. Lett. 80, 3288 (1998).

${ }^{50}$ F. Faupel, P. W. Hüppe, and K. Rätzke, Phys. Rev. Lett. 65, 1219 (1990).

${ }^{51}$ G. Rummel and H. Mehrer, Phys. Status Solidi A 185, 327 (1994).

${ }^{52}$ K. Rätzke and F. Faupel, J. Non-Cryst. Solids 181, 261 (1995).

${ }^{53} \mathrm{H}$. Wipf, in Hydrogen in Metals III, Vol. 73 of Topics in Applied Physics, edited by H. Wipf (Springer-Verlag, Berlin, 1997), p. 51.

${ }^{54}$ K. R. A. Heesemann, F. Faupel, J. Hoffmann, and K. Heinemann, Europhys. Lett. 29, 221 (1995).

${ }^{55}$ K. Rätzke, P. W. Hüppe, and F. Faupel, Phys. Rev. Lett. 68, 2347 (1992).

${ }^{56}$ D. Caprion, Ph.D. thesis, Université Montpellier II, 1998.

${ }^{57}$ S. D. Bembenek and B. B. Laird, Phys. Rev. Lett. 74, 936 (1995).

${ }^{58}$ P. Jund, D. Caprion, and R. Jullien, Europhys. Lett. 37, 547 (1997).

${ }^{59}$ P. Jund, D. Caprion, and R. Jullien, Phys. Rev. Lett. 79, 91 (1997).

${ }^{60}$ P. Ehrhart, K. H. Robrock, and H. R. Schober, in Physics of Radiation Defects in Crystals, edited by R. A. Johnson and A. N. Orlov (North-Holland, Amsterdam, 1986), p. 3.

${ }^{61}$ W. G. Hoover, S. G. Gray, and K. W. Johnson, J. Chem. Phys. 55, 1129 (1971).

${ }^{62}$ B. B. Laird and A. D. J. Haymet, Mol. Phys. 75, 71 (1992).

${ }^{63}$ W. G. Hoover, D. A. Young, and R. Grover, J. Chem. Phys. 56, 2207 (1972).

${ }^{64}$ W. C. Swope, H. C. Andersen, P. Berens, and K. R. Wilson, J. Chem. Phys. 76, 637 (1982).

${ }^{65}$ R. Fletcher and C. M. Reeves, Comput. J. (UK) 7, 149 (1964).

${ }^{66}$ T. A. Weber and F. H. Stillinger, Phys. Rev. B 31, 1954 (1985).

${ }^{67}$ F. Yonezawa, J. Non-Cryst. Solids 35/36, 29 (1980).

${ }^{68} \mathrm{U}$. Balucani and M. Zoppi, Dynamics of the Liquid State (Clarendon, Oxford, 1994).

${ }^{69}$ A. Rahman, Phys. Rev. 136, A405 (1964).

${ }^{70}$ R. Zorn, Phys. Rev. B 55, 6249 (1997).

${ }^{71}$ A. Heuer, in Microscopic View of Low-Temperature Anomalies in Glasses, edited by P. D. Esquinazi (Springer, Berlin, 1998).

${ }^{72}$ C. Oligschleger, Ph.D. thesis, RWTH Aachen, 1994.

${ }^{73}$ H. R. Schober, C. Gaukel, and C. Oligschleger, Prog. Theor. Phys. Suppl. 126, 67 (1997).

${ }^{74}$ B. Bernu, J. P. Hansen, Y. Hiwatari, and G. Pastore, Phys. Rev. A 36, 4891 (1987). 